\title{
On the Applications of Efficient NFV Management Towards 5G Networking
}

\author{
Ioannis Giannoulakis*, Emmanouil Kafetzakis*, George Xylouris*, George Gardikis* and Anastasios Kourtis* \\ *Institute of Informatics \& Telecommunications, \\ National Centre for Scientific Research "Demokritos", \\ P.O. Box 60228, GR-15310 Ag. Paraskevi, Greece. \\ Email: \{giannoul; mkafetz; xilouris; gardikis; kourtis\}@iit.demokritos.gr
}

\begin{abstract}
The paper leverages the emerging paradigm of Network Function Virtualization (NFV) in an attempt to outline from the network management point of view the current trends on the road to $5 \mathrm{G}$ region. To that end, relevant architectural frameworks and concepts have been presented and the anticipated network densification through the notion of Edge Cloud has been discussed. Within this context, added value assets that can be generated by following some of the analysed principles have been also included. Finally, open issues and challenges with respect to the NFV functionalities and their materialization according to 5G requirements have been elaborated and some key new concepts about network and small cell virtualization have been introduced.
\end{abstract}

\section{INTRODUCTION}

Modern telecommunication society becomes a witness of the convergence of cloud networking, fast connectivity and high processing power taking place over the existing Internet model. However, in order to turn page and introduce the long anticipated 5G model, i.e., a next generation framework for omnipresent, ultra-low latency broadband infrastructure capable of providing the critical and highly demanding applications and services of the future Internet, novel ideas and approaches are required. The fresh, groundbreaking architecture will require revolutionary changes in both network infrastructure and modeling, where user equipment should be jointly integrated and optimised within the surrounding context. We expect that the majority of further improvements in the field will come from achievements that will leverage both important achievements in networking technologies, like those proposed by the NFV architecture [1], as well as open software platforms for network management.

In $4 \mathrm{G}$ systems, residential or enterprise femtocells solved partially the capacity crunch problem through spectrum reuse. Following the same trend, in the next years we expect hyper-dense outdoor small cell deployments for achieving increased bandwidth, low latency, low power consumption and cost-effectiveness. Apart from reusing spectrum, this will be achieved by the rapid convergence of the Telecommunication and the Information Technology (IT) worlds. More specifically, it is foreseen that both communication and computing/storage capabilities will have to be integrated into the small cells.

Future operator ecosystems will be consisted by a combination of centralized clouds on IT infrastructure as well as smaller-scale cloud platforms at the edge, towards enhanced capabilities, like edge caching and processing, for demanding applications. In this context, already envisaged small cell managed services, where operator companies and customers are supplied with mobile coverage and capacity to offer or to consume, depending on which side they belong, can be further enhanced by the innovations following NFV notion.

In the industrial field, a number of initiatives and platforms have emerged that align themselves to the ETSI NFV proposed architecture. Notably, Alcatel-Lucent's Cloudband platform, aims at transforming carrier-grade service provider networks with distributed footprint into a single, manageable, virtual cloud [2]. Hewlett-Packard, via the OpenNFV platform [3], proposes an open reference architecture, encompassing a service portfolio, enforced by an ecosystem of International Standard Versions and application developers. A reference architecture based on the ETSI NFV principles is jointly proposed by Tieto, Intel and Qosmos [4]. The proposed architecture is also implemented in the frame of an ETSI NFV Proof of Concept [5]. This work provides additionally a mapping of the architecture to the currently available software components. Finally, CloudNFV is deploying a mixture of Virtual Network Functions (VNFs), cloud application components, real network devices and multi-operator federated services over an architecture aligned to ETSI [6].

Likewise, in the research field numerous funded proposals have proposed their architectures either aligned or relevant to the ETSI NFV work. With the aim of promoting the NFV concept, the European FP7 project T-NOVA [7] described briefly in the next section, introduces a novel enabling framework, allowing operators not only to deploy VNFs for their own needs, but also, to offer them to their customers as valueadded services. Project UNIFY [8] proposes an architecture for increasing the potential of virtualization and automation across the whole networking and cloud infrastructure, including core and datacenter networks. Project MCN [9] approaches the integration between the cloud and the telecommunications worlds, making operators benefit from the principles of virtualizing network resources.

The purpose of this paper is to present an overview of current trends in the network management area towards the $5 \mathrm{G}$ framework and to identify potential novel scenarios and open issues on the field. Building upon the emerging NFV 
concept, we argue that a rethinking towards the Edge Cloud as a remedy for network densification could be beneficial for the traditional and virtual telecom operators and to the endusers as well.

This paper is structured as follows. Section II presents the basic NFV notions and introduces the major aspects of the relevant T-NOVA project. Section III treates the way network densification can be achieved under the scope of $5 \mathrm{G}$ networks, while Section IV discusses some of the related open issues. Finally, Section V concludes the paper.

\section{NFV DEPLOYMENT IN THE CORE NETWORK}

\section{A. NFV Advantages}

NFV Industry Specication Group (ISG) of ETSI is currently the prominent industry-led initiative in the NFV area. According to NFV reference framework, the architecture of service providing within a network is transferred to a diverse scene of functions, based on a virtual/cloud framework. NFV can support the abstraction of functions related to networking procedures from hardware to software through the employment of a 'hypervisor level' and allows the agile distribution of operations throughout the locations of interest, i.e., network nodes, data centres and so on. Moreover, a wide range of the operational modules are in scope, like routing, switching, load balancing and security elements, e.g., deep packet inspection and firewalling.

The NFV concept encompasses several critical benefits when it is applied in the networking domain as a substitute to the ordinary execution of the in-network functionalities through stand-alone hardware devices. To start with, consolidating the hardware resources yields reduced costs for acquiring and maintaining equipment, as well as energy expenditures cutoffs. NFV allows the sharing of network resources between various Network Functions and users in a far more easy and adaptive manner, while resources assigned to each function can be rescaled dynamically. Therefore, VNFs can be tailored according to the precise requirements, in order to accommodate and handle efficiently all relevant connectivity components. We should note also that NFV accounts for faster and more convenient deployment of Network Functions at severely reduced risk and cost. This property suggests an important reduction of the time that new solutions require to reach the market and also, that novel services even in experimental level can 'live' together with those that are already in use. Thus, innovation on the field is further supported by opening a new part of the market, in order to involve exclusively software developing actors, i.e., academia, small and medium-sized enterprises and so on.

The path of building the network infrastructure by applying exclusively hardware-based elements has worked well for some time, carrying out most of the network functionalities through separate appliances. In the NFV concept, Network Functions can provide to operators resilience and agility through the 'service chaining' model, (see, e.g., Figure 1). A VNF chain can be created and updated in a dynamic and

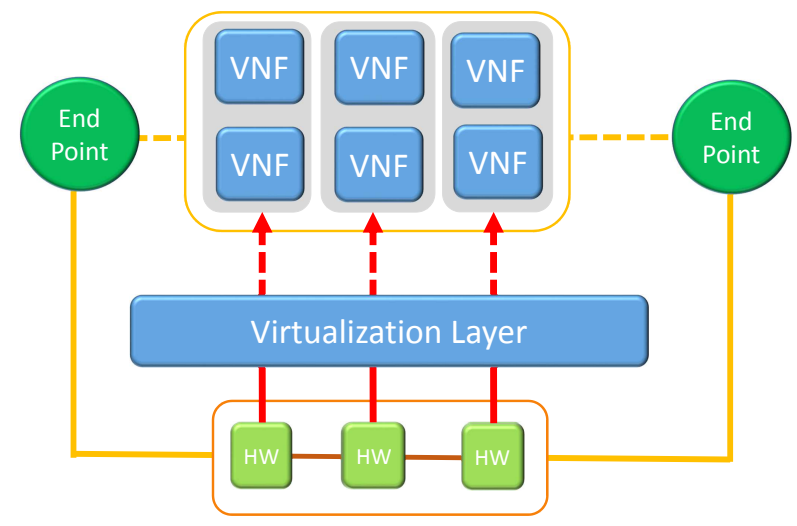

Fig. 1. End-to-end network service virtualization

active manner. Hence, VNFs can operate in a more complex way, following the most applicable path towards their target.

\section{B. T-NOVA Architecture}

The currently running T-NOVA project aims to promote the NFV concept through the design and the implementation of a framework for the provisioning, monitoring and management of VNFs over Network/IT infrastructures. Its objective is to provide composite services in the form of Network Functions as a Service (NFaaS), by contributing both connectivity services (i.e. connectivity established even through virtual networks if required, based on Software Defined Networking (SDN) and a set of associated Network Functions according to the users' needs. As a consequence, novel management schemes need to be developed with respect to a variety of operations, as e.g., resource matching, provision and adaptation in real time, within the orchestrator's platform.

Within the context of T-NOVA, a new enabling schema is introduced (see, e.g., Figure 2). It gives the chance to network operators not only to deploy VNFs for their own needs at will, but also to offer them to third party customers as 'addedvalue' services. Moreover, the aim is to provide VNFs (e.g., firewalls, routers, gateways and so on) on-demand and as-aService, thus wiping out the requirement for obtaining and sustaining expensive and specialized hardware at user's place. For this purpose, an innovative "Network Function store" is established, following the already successful concept of Operation System-specific "Application Stores", and a novel "brokerage platform" that permits interaction of users with the T-NOVA service providers or third party developers, is being constituted.

With respect to the proposed functionalities and processes, the high-level architecture can be epitomized in a vertical layered structure as follows. At the bottom exists the NFV Infrastructure (NFVI) layer that includes the physical and virtual nodes (e.g., storage systems, switches, routers, commodity servers etc.) on which the services are deployed. On top of NFVI, the NFV Infrastructure Management layer is placed, 


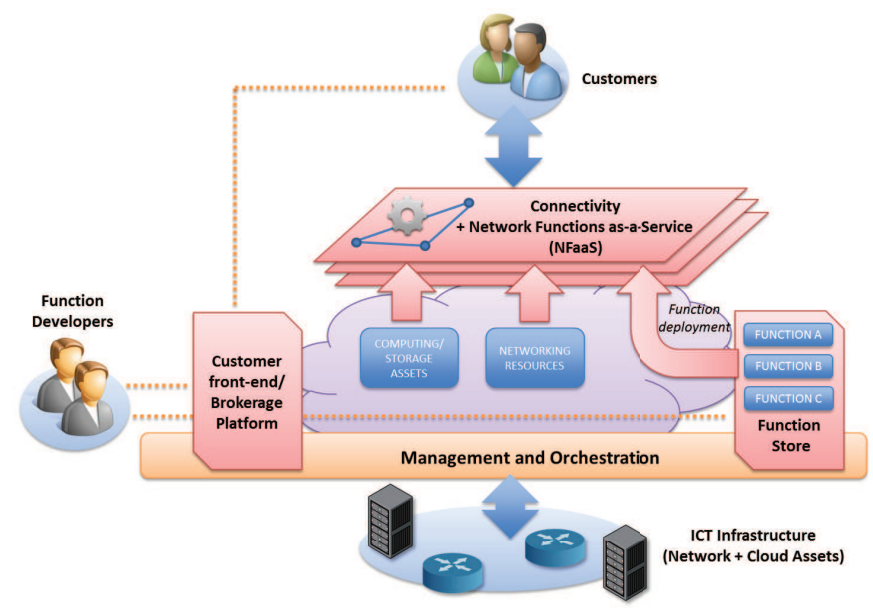

Fig. 2. T-NOVA high-level architecture

which includes the infrastructure management entities. On top of this resides the orchestration layer where the T-NOVA Orchestrator is based and also where the Network Function store is included. Finally, the 'marketplace' layer contains all the customer-facing modules, which facilitate multi-actor involvement and implement business-related functionalities.

To that end, building upon NFV concepts as they are elaborated within T-NOVA as well as other relevant projects, we believe that the virtualization of $5 \mathrm{G}$ critical infrastructure elements, like e.g., small cells, will bring added value to all actors involved in the scene, including operators and end-users. More specifically, we envision the 'liquidation' of small cell resources and their utilization as autonomous virtual networks (e.g. in the form of a 'network slices') with embedded on-demand capabilities, according to the operators' profiles. Such functionalities create an opportunity to rethink how future networks (i.e. 5G and beyond) will be constructed and operated at their entirety.

\section{App Coverage through Network Densification AND MOBILE EDGE CLOUd}

Most European telecom operators are in the beginning of a long capital refresh cycle, gradually planning their future upgrades from Long Term Evolution (LTE)/LTE-Advanced. Despite the strict performance requirements of $5 \mathrm{G}$ era (i.e., ultra-high broadband links, support of billions of interconnected devices, low latency for tactile internet, low power consumption, etc.), the direction that will be adopted needs to avoid platform fragmentation and to be compatible with existing technology enablers and management schemes. NFV technique is deployed on standardized virtualized hardware for reducing the cost of ownership, mainly in the core network. The list of already implemented virtualized mobile network function applications includes the Evolved Packet Core (EPC), the IP Multimedia Subsystem (IMS) and the Radio Access Network (RAN) part. NFV adoption improves significantly core network efficiency and allows easy testing of innovative architectures and fast service deployment. To the best of our knowledge, NFV support has not yet propagated to small cell platforms i.e. there do not exist commercial small cells that can host VNFs in the market. However, such an evolution is expected in the short term, since the impact of virtualized network appliances running at the network edge is expected to rise. Such an evolution implies the upgrade of small cell platforms from plain connectivity bridges to NFVI-Points-ofPresence (NFVI-PoPs, in ETSI NFV ISG terminology), i.e., virtualized clusters able to accommodate VNFs.

For example, a candidate use case of NFV application on small cells could be small cell caching (dynamic cache VNF deployment on NFV-enabled small cells), especially if caches are updated during LTE-network off-peak times. Having distributed storage capacity and optimal content placement to the wireless segment is anticipated to reduce drastically the latency in 5G networks. In a similar way, edge Domain Name System (DNS) caches can be configured to cache the DNS content of central servers. In another direction, for data traffic characterization, monitoring and policing reasons, secure gateways (SEC GWs) can be launched as VNFs hosted in small cells. Today, data traffic is often encrypted through Internet Protocol Security (IPSEC) between the eNodeB and the SEC GW in the core network (only the Differentiated Services Code Point (DSCP) is visible in the outer Internet Protocol (IP) header). By launching localized SEC GWs, a module could decrypt the traffic and look all additional information (e.g., bearer identity) or even run a Deep Packet Inspection (DPI) VNF in order to proceed to traffic differentiation and content-aware techniques. Finally, since high quality video is expected to be the prime driver of demand in the coming years, all functions that are related to LTE Broadcast functionality are of high interest (especially the virtualization of evolved Multimedia Broadcast Multicast Service (eMBMS) that intends to deliver high quality video in a spectrally efficient way).

The unified management of the hyper-dense small cell network and the various VNFs at the edge is a great challenge. First of all, it requires auto-integration and self-management capabilities from the small cell (beyond today's self-organizing network features), which have to be reflected in the architectural layer to achieve their full potential. Starting from the description model of the lightweight computing clusters across the network infrastructure, and continuing with the study of placement of VNFs, the Virtual Infrastructure Manager (VIM) implementation and the design of the overall orchestrator can be considered the roadmap towards the small cell "cloudification".

Figure 3 presents the different NFV management entities, i.e., the VIMs and the Orchestrator that control the NFVenabled small cells. Despite the scarcity of the spectrum, the autonomy of small cells (provided by design) will allow zero touch deployment of large scale of devices. However, the VIM should be able to add and remove resources (computing/storage or 'slices' of network bandwidth) in selected small cell/VNF PoP, according to the needs and according the Network Function demands. To achieve this, according 


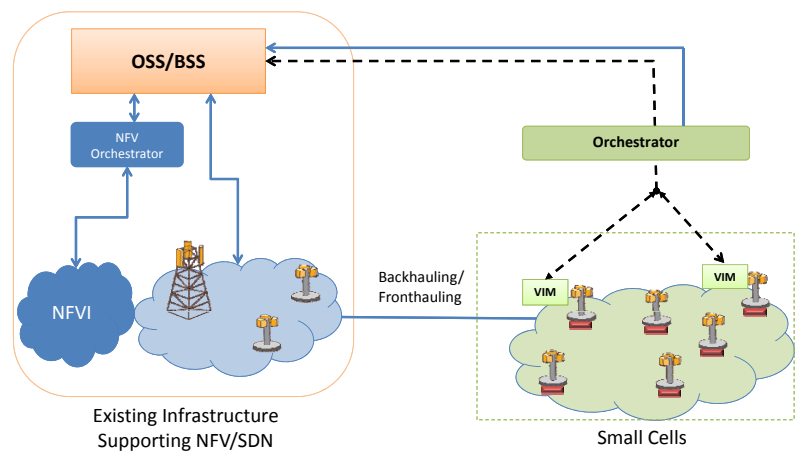

Fig. 3. NFV enabled small cells

to the contracted Service Level Agreement, the VNF specific requirements have to be mapped to the required virtualised infrastructure assets (e.g., virtual and physical machines, storage and networking elements, etc). The mapping procedures should also consider the network topology, connectivity and network Quality of Service constraints, as well as function characteristics (e.g. some functions may require low delay, low error rate, high bandwidth and so on). Since virtualised resources can be centralised in a single NFVI-PoP or distributed across several edge NFVI-PoPs, the management and orchestration entities will also have to decide on the most appropriated NFVI-PoPs (core or edge) to deploy the function.

Apart from the VNFs and the small cell/NFVI-PoPs management challenges, new and more complex network services will be provided based on the combination/chaining of several VNFs. Therefore, in addition to the management and orchestration of the VNFs and of the associated NFVI-PoP, the respective procedures have to be defined also at the service level. These will coordinate several VNFs, as well as their association. Moreover, the role of the Orchestrator will be to allow coordination between VIMs and to make all components of the 5G ecosystem operate optimally. Given the massive mobility of users and devices in $5 \mathrm{G}$ platforms, cognitive management features should be exploited by the Orchestrator, in order to deploy VNFs and to forward traffic according the function chaining graph. All management decisions should be transparent to the end users and should optimize the finally perceived Quality of Experience.

\section{UpComing Challenges FOR NFV-Framed 5G NETWORKING}

A thorough analysis on the abstraction of network services, the respective management aspects and some open issues has been presented in [10]. Network functionalities on classical systems like e.g., performance management, error handling, security and so on, are normally governed in a centralized way and they are distinguished for their intense complexity. Many of these components require specialized treatment, and frequently this occurs through an interaction with a human, particularly due to the explosion in the number of network

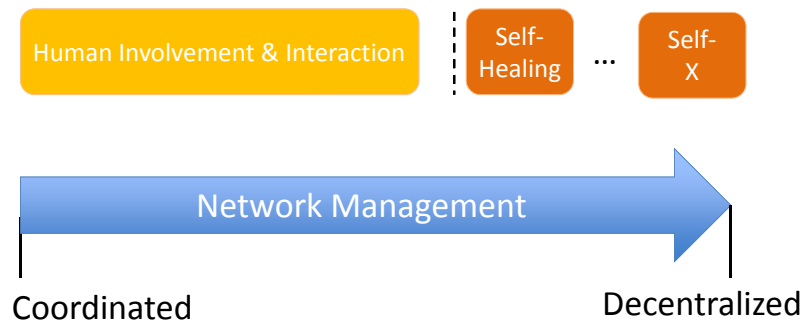

Fig. 4. Evolution towards Self-X attributes

devices and applications. Clearly, in order to serve future needs, the network archetype needs to shift towards a fully automated ecosystem, offering ubiquitous and immediate connectivity. On the other hand, there exists the concession that decisions related to control and networks management should be at most distributed in order to take advantage of the low response times. Not only will such an evolution increase the network's availability, but it will reduce the costs related to its control and operation as well.

For now, although many attempts have been targeting decentralized solutions, (see, e.g., ETSI actions for Autonomic Future Internet [11] and efforts on the Self-Organizing Networks [12]) and 'real-time' network operations, still combinations of concentrated and decentralized solutions (also known as 'autonomic') are mainly adopted. The long anticipated Self$\mathrm{X}$ features, i.e., Self-Coordination, Self-Planning, Self-Healing and so on, are based exactly on this type of selected management. Therefore, important attempts are targeted, and should continue to do so, towards fully automated solutions (see, e.g., Figure 4).

Also, since one of the principal purposes of virtualization is efficiency, hypervisors associated with sophisticated systems evolve at a great pace and consist one of the critical factors for optimizing performance and utilization. Emerging VNF chaining mechanisms, enable the mapping of Network Functions to the virtualized environment and therefore, are an appealing option for data centres and cloud computing. Furthermore in this direction, modification of hypervisors to support hardware accelerators becomes a key enabler in order to support heavy processing workloads as e.g., the demanding LTE eMBMS and applications like Ultra-High-Definition video (as we envisage that it will be the killer application for 5G networking).

Inside the virtualized universe it is difficult for someone to apply the traditional test and measurement methods. Instead, measurement software applications should be deployed. The implementation and configuration of connections between Virtual Machines within a SDN environment is typically undertaken by SDN controllers, like e.g., OpenDaylight, and through the OpenFlow protocol that manages both physical and virtual switches. Nevertheless, in a real-world system, the hardware interconnecting the various parts still plays the major role and hence, any monitoring system should be able to take measurements in both the virtual and physical domain. 
Estimating performance measures about traffic transmitted among the devices of the physical network, as well as between a number of VNFs, like e.g., deep packet inspectors, firewalls, load balancers, routers and so on, remains a difficult yet important issue within SDN/NFV environment.

A final issue to mention has to do with the description of the market model and the willingness of end users to pay for the forthcoming $5 \mathrm{G}$ functionalities. To that end, one should not only define the importance of NFV in reducing CAPEX and OPEX, but also, a concrete interpretation of who will be the actors and who will shoulder the cost of network ownership in the new era, are required.

\section{Conclusions}

Evidently, industry, academia and research projects active in the area of network management, are paying increasing attention to the evolutions in the area of NFV. They strongly align their attitude towards including not only separate aspects, but the entire NFV architectural framework in their positioning for $5 \mathrm{G}$ race. At the same time, they extend and diversify in areas heavily related to network densification, in order to accommodate their intended business cases.

In this context, one of the forthcoming challenges is to develop a novel integrated framework that will allow the deployment and the intelligent management of NFV-enabled small cells, enriched with the ability to provide IT resources at the network's edge. To that end, several benefits will arise, provisioning of connectivity services on demand increased system scalability and improved network coverage to name a few.

On the other hand, each solution proposed in the grounds of NFV needs to be compatible with the existing network management infrastructure, in order to allow a smooth transfer path, towards a fully virtualised infrastructure. Also, when the conversation comes to large-scale operational field, scalability and efficiency should remain in scope, keeping at the same time alive the tight bond between IT domain and networking resources.

\section{ACKNOWLEDGMENTS}

The work carried out within this manuscript has been partially funded by the European Commission through the IST FP7 619520 T-NOVA project. We thank all our partners for their important and helpful contributions.

\section{REFERENCES}

[1] ETSI, "Network Functions Virtualisation (NFV): Architectural Framework," ETSI GS NFV, vol. 2, p. v1, 2013.

[2] CloudBand-Alcatel/Lucent, www.alcatel-lucent.com/solutions/ cloudband.

[3] "Open Platform for NFV," www.opnfv.org.

[4] Qosmos, Tieto, and Intel, "Realising the Benefits of Network Functions Virtualisation in Telecoms Networks," http://embedded.communities. intel/com/docs/DOC-7981.

[5] "GS NFV-PER 002 v1.1.1. NFV Proof of Concepts framework," May 2013.

[6] "GS NFV 001 Network Function Virtualisation (NFV) - Use Cases," 2013.

7] T-NOVA, "FP7-ICT - T-NOVA," www.t-nova.eu.

[8] UNIFY, "FP7-ICT-UNIFY," https://www.fp7-unify.eu.

[9] MCN, "Deliverable D2.2 - Overall Architecture Definition."

[10] S. Kuklinski and P. Chemouil, "Network Management Challenges in Software-Defined Networks." 2014, pp. 2-9.

[11] "ETSI GS AFI 001 v1.1.1," http://www.etsi.org/deliver/etsi_gs/AFI/001_ 099/001/01.01.01_60/gs_afi001v010101p.pdf.

[12] “3GPP TS 32.500, Self-Organizing Networks (SON): Concepts and Requirements (Release 9),” 2009. 